\title{
3D Cardiac Anatomy Reconstruction Using High Resolution CT Data
}

\author{
Ting Chen ${ }^{1}$, Dimitris Metaxas ${ }^{1}$, and Leon $\mathrm{Axel}^{2}$ \\ 1 Rutgers, the State University of New Jersey, Piscataway, NJ 08854, USA, \\ chenting@gradphics.cis.upenn.edu, \\ dnm@cs .rutgers .edu \\ 2 New York University, School of Medicine, 550 first Avenue, New York, NY, 10016, \\ USA, \\ leon.axel@med.nyu.edu
}

\begin{abstract}
Recent advances in CT technology have allowed the development of systems with multiple rows of detectors and rapid rotation. These new imaging systems have permitted the acquisition of high resolution, spatially registered, and cardiac gated 3D heart data. In this paper, we present a framework that makes use of these data to reconstruct the 3D cardiac anatomy with resolutions that were not previously possible. We use an improved 3D hybrid segmentation framework which integrates Gibbs prior models, deformable models, and the marching cubes method to achieve a sub-pixel accuracy of the reconstruction of cardiac objects. To improve the convergence at concavities on the object surface, we introduce a new type of external force, which we call the scalar gradient. The scalar gradient is derived from a gray level edge map using local configuration information and can help the deformable models converge into deep concavities on object's surface. The 3D segmentation and reconstruction have been conducted on 8 high quality CT data sets. Important features, such as the structure of papillary muscles, have been well captured, which may lead to a new understanding of the cardiac anatomy and function. All experimental results have been evaluated by clinical experts and the validation shows the method has a very strong performance.
\end{abstract}

\section{Introduction}

Cardiovascular disease is the most common cause of death in America and there is a strong need to detect and diagnose such disease in its early stages. The automated analysis of cardiac images has been improved dramatically in the past few year and provides a way to get detailed anatomic and functional information of the heart. One of the pre-requisites of quantitative analysis of cardiac images is the accurate location of the surfaces of the ventricles and the myocardium. The segmentation and the subsequent reconstruction of the ventricular-myocardium surface is not trivial because of the noise, blurring effects at edges, and motion artifacts. In addition, the segmentation should be automated and time-efficient in order to be clinically applicable. 
Recently, several methodologies have been proposed by different research groups to solve the cardiac segmentation problem. In [8], 9] deformable models are integrated with statistical models or atlases to enable the use of both intensity and spatial information. Several techniques derived from the Active Shape Model (ASM) 4] and the Active Appearance Model (AAM) 7] have been used in [10] to improve the reliability and consistency of the segmentation process. However, all of these approaches aim to solve 2D problems, so that the segmentation and reconstruction may miss important features distributed along the cardiac long axis.

In [5], a 3D ventricular surface is constructed using a deformable model from tagged MRI data. This approach has succeeded in capturing the data related to the strain and stress distribution on the surface of the myocardium. However, the reconstructions, especially of the endocardial surface, are limited by the sparsity of the data and the low quality of the shape model. Important anatomic structures, such as the papillary muscles and valves, can be missed in this model. In [1] a 3D statistical model for atria and ventricles has been built using a 3D AAM. However, the deformable model does not converge well to the data at concavities and convexities on the ventricular surface because of: 1) the smoothing effect of the deformable surface at concavities, and 2) the lack of corresponding details in the initialization of the deformable model.

In [6], the Gradient Vector Flow approach has been proposed to address the problem of deformable model convergence at concavities. The use of the Gradient Vector Flow can extend the attraction range of the gradient information by diffusing a gray level or binary edge map. The extended gradient flow enables the deformable model to fit into concavities on the object surface. However, the computation process of the Gradient Vector Flow is tedious. Moreover, the Gradient Vector Flow's performance degrades as the concavity becomes deeper.

In previous work [2], we have proposed a 3D hybrid framework that is capable of achieving sub-pixel accuracy segmentation of medical images. In this paper, we improve the framework by using a new type of external force during model deformation, which derives from the idea of "scale" in 3. The new external force has a superior performance in concavity convergence.

The remaining parts of this paper are organized as follows. In section 2, we present the acquisition of the high quality CT data. In section 3, the hybrid framework will be explained. Related improvements, such as the definition of the new external force, will also be given. Section 4 shows some representative high quality 3D reconstructions of cardiac objects produced by our framework. We will also discuss an interesting anatomical structure based on the 3D reconstruction result. Section 5 presents the validation results and the final conclusion.

\section{Data Acquisition}

Image acquisition with multi-detector CT (MDCT) with continuous table motion is accomplished using cardiac gating. Current MDCT systems permit coverage of the chest in a breath hold, with effective temporal resolution on the order of 
$120 \mathrm{~ms}$ of the cardiac cycle and isotropic spatial resolution in three dimensions on the order of $0.75 \mathrm{~mm}$; a 16 detector-row CT system (Siemens Sensation 16, SMS, Malvern, PA) with these specifications was used to acquire the images used in this study. The images were acquired during the intravenous infusion of radiographic contrast agent (Visipaque 320) at $4 \mathrm{ml} / \mathrm{s}$; image acquisition was timed to coincide with the maximum opacification of the cardiac chambers. The heart rate was slowed using beta blockers, preferably to 60 BPM or less, in order to minimize motion blurring; image acquisition was gated from mid to late diastole to minimize motion blurring and to capture the ventricles in a relatively relaxed state. The data created have 512 by 512 by 281 pixels.

\section{Methodology}

The hybrid framework proposed in 2 consists of 4 internal modules: the region segmentation module driven by high order Gibbs prior models, the deformable model, marching cubes, and the parameter updating module.

The acquired high quality CT data (in DICOM format, refer to section 2) are translated into raw data sets using the image reader provided by Insight Toolkit (ITK) for further processing.

During the segmentation process, we first apply the Gibbs prior models onto the image. The joint distribution of the medical image is expressed in the following Gibbsian form:

$$
\Pi(X)=Z^{-1} \exp (-H(X))
$$

where we use $\mathbf{X}$ to denote the set of all possible configurations of the image $X, z$ is an image in the set of $\mathbf{X}, Z=\sum_{z \in \mathbf{X}} \exp (-H(z))$ is a normalizing factor, and $H(X)$ is the energy function of image $X$. The image is segmented by constructing a suitable energy function for the image and minimizing it. A Gibbs prior energy is defined as follows:

$$
H_{\text {prior }}(X)=H_{1}(X)+H_{2}(X)
$$

where $H_{1}(X)$ models the piecewise pixel homogeneity statistics and $H_{2}(X)$ models the object boundary continuity. In studying the CT data, we find that the ventricular-myocardium surfaces are not smooth; instead, the surface features many small concavities and convexities. In addition, the existence of the papillary muscle and cardiac valves makes the surface even more irregular. In our implementation, such surface features have been modelled by assigning low potential to local configurations that ensemble a continuous surface with angles so that during the energy minimization the Gibbs model is capable of capturing the saw-like cardiac chamber surface structure.

According to Bayesian framework, the final energy function we minimized is a posterior energy which is in the form of:

$$
H_{\text {posterior }}(X, Y)=H_{\text {prior }}(X)+H_{\text {observation }}(X, Y)
$$


where $H_{\text {observation }}(X, Y)=\vartheta_{3} \sum_{s \in X}\left(y_{s}-x_{s}\right)^{2}$ if we assume the image has been distorted by Gaussian noise, $y_{s}$ is the observation of pixel $s, x_{s}$ is the estimated value, $\vartheta_{3}=2 \sigma^{2}$ is the weight for the constraint of observation, and $\sigma$ is the standard deviation.

The $3 \mathrm{D}$ binary mask output by the region segmentation module is used as the input to the marching cubes module. A 3D mesh consisting of triangular elements is created along the surface of the binary mask. The 3D mesh is used as the initial geometry of the deformable model.

We use Langrange dynamics to deform our model as follows:

$$
\dot{\mathbf{d}}+\mathbf{K d}=\mathbf{f}_{e x t}
$$

where $\mathbf{d}$ is the displacement, $\mathbf{K}$ is the stiffness matrix, and $f_{\text {ext }}$ are the external forces.

The deformable model deforms under the effect of the internal force $\mathbf{K d}$ and external forces $\mathbf{f}_{\text {ext }}$. The internal forces keep the deformable model surface smooth and continuous during its deformation. The external force will lead the model to the object surface using image information such as the gradient. In [2], the external force is derived from the second order derivative of a diffused gradient map. In this paper, we describe a new type of external force that will improve the convergence at concavities (refer to section 3.1).

To solve equation (4) we use the Euler integration as follows:

$$
\mathbf{d}_{n e w}=\dot{\mathbf{d}} \cdot \Delta t+\mathbf{d}_{\text {old }}
$$

where $\Delta t$ is the time step. The deformation stops when the force equilibrate or vanish.

The deformable model segmentation will then be used to update the parameters of the Gibbs prior model, which includes mean intensity, standard deviation, and the local potentials (refer to [2]). Then we will start a new loop of the segmentation framework. In most cases, we can achieve a good segmentation result within 2 iterations.

We show the process of our segmentation-reconstruction method in figure 1.

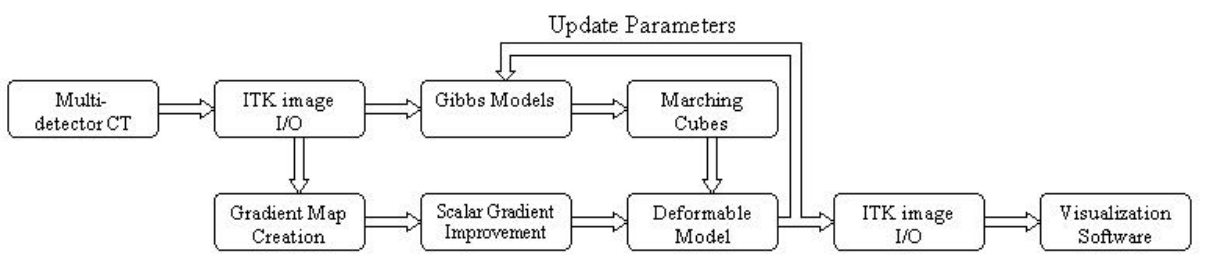

Fig. 1. Hybrid Segmentation Framework 


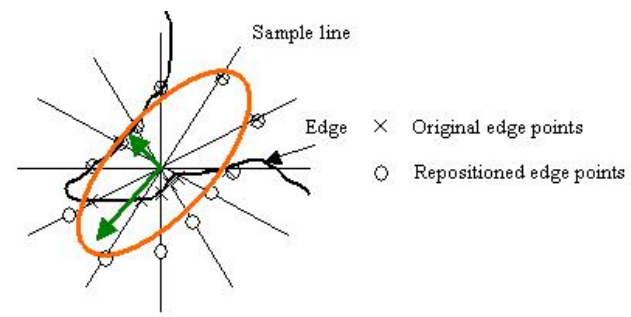

Fig. 2. 2D scalar gradient vector construction

\subsection{Scalar Gradient}

During the process of fitting the deformable model to the cardiac surface, we use a new type of external force, which we call the 'scalar gradient', to achieve better convergence at concavities. The scalar gradient is derived from the definition of 'tensor scale' in [3]. The tensor scale at an image pixel is the parametric representation of the largest ellipse (for 2D) centered at the pixel and containing only the homogeneous region under prior criterion. It contains information of the orientation, anisotropy, and thickness of local structures.

In a $2 \mathrm{D}$ implementation, to calculate the scale at a pixel, we first find the closest edge points to the pixel along a group of sample lines that are normally distributed over the entire angular space around the pixel. Different from the computation of the tensor scale, for each conjugate pair of sample lines, we reposition the sampled edge points by selecting the point that is further from the pixel and reflect it on its complementary sample line. Let the coordinates of the edge points on sample lines be $\left(x_{1}, y_{1}\right),\left(x_{2}, y_{2}\right), \ldots,\left(x_{2 m}, y_{2 m}\right)$, and that of the pixel be $\left(x_{0}, y_{0}\right)$; we can compute the local covariance matrix $\mathbf{M}$, where

$$
\begin{gathered}
\mathbf{M}_{1,1}=\frac{1}{2 m} \sum_{i=1,2, \ldots, 2 m}\left(x_{i}-x_{0}\right)^{2}, \mathbf{M}_{2,2}=\frac{1}{2 m} \sum_{i=1,2, \ldots, 2 m}\left(y_{i}-y_{0}\right)^{2} \\
\mathbf{M}_{1,2}=\mathbf{M}_{2,1}=\frac{1}{2 m} \sum_{i=1,2, \ldots, 2 m}\left(x_{i}-x_{0}\right)\left(y_{i}-y_{0}\right)
\end{gathered}
$$

$\mathbf{M}$ is a symmetric matrix with positive diagonal elements so it has two orthogonal eigenvectors and two positive eigenvalues $\lambda_{1}, \lambda_{2}$ associated with the eigenvectors. We use the direction of the principal radius as the direction of the scalar gradient vector, and the magnitude of the scalar gradient is proportional to $\frac{\lambda_{1}}{\lambda_{2}}$, where $\lambda_{1}>\lambda_{2}$. The computation of the 2D scalar gradient is shown in figure 2 , and it is simple to extend it into $3 \mathrm{D}$.

For simplicity, we derive the scalar gradient field using the edge map of the original image. The threshold of the edge can be calculated using the result of the Gibbs segmentation. This map will be used to locate those edge points around pixels during the computation of local ellipses. We then combine the scalar gradient with the original gradient flow to form the external force field. 


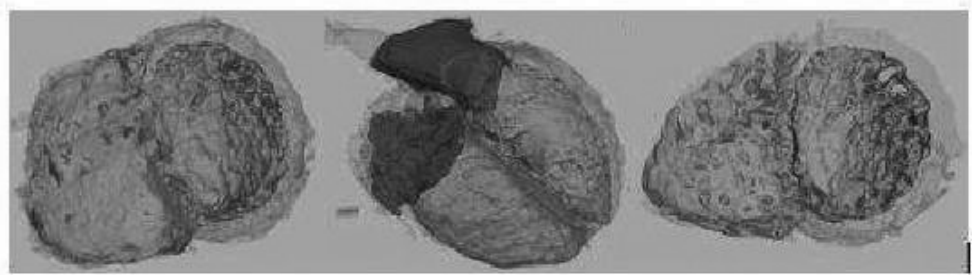

a

b

c

Fig. 3. We show the LV and RV atria in blue, the ventricles in green, and the epicardial surface in transparent red. a) shows a view of the heart from the bottom. b) shows a view of the heart from the front. In c) we intersect the $3 \mathrm{D}$ reconstruction using a plane that is vertical to the long axis of the heart and show the resulting endocardial and epicardial surface from the top.

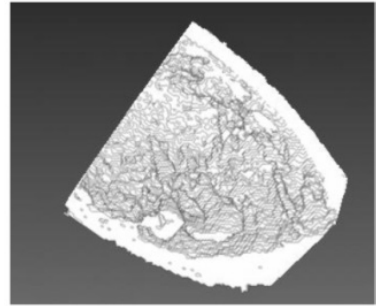

(a)

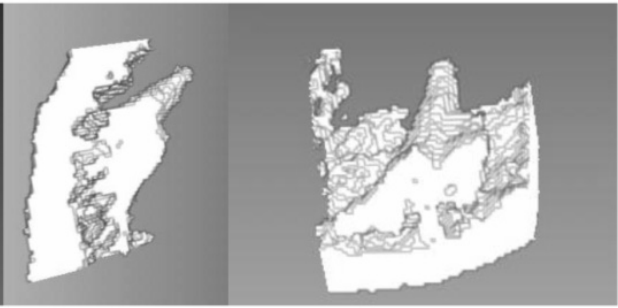

(b) (c)

Fig. 4. Anatomic structure of the papillary muscle. 3 views of the papillary muscle being cut by user-defined planes. The white plane is the intersection of cutting plane to the $3 \mathrm{D}$ reconstruction of myocardium. Notice that there are tunnels for blood between the papillary muscle and the myocardium.

\section{Experimental Results and Discussion}

We applied our method to eight different cardiac datasets. In figure 3, we show the $3 \mathrm{D}$ reconstructions of cardiac surfaces based on the segmentation result of one dataset. The data size is 256 by 256 by 140 . Our segmentation method has succeeded in capturing the surface features and the global shape of the cardiac chambers. The whole segmentation process takes about 10 minutes on a P4 2.2G Hz desktop.

Figure 4 illustrates the anatomic structure of the papillary muscle. The rendered 3D images of the segmented heart wall show the structures of the trabeculae carnae and papillary muscles very clearly on the endocardial surface of the ventricular cavity. The three-dimensional nature of these structures and their anatomical relationships are much more readily appreciated with such a rendering than from the original image data, even with interactive reformatting of the displayed image plane. In particular, it can be appreciated how the rope-like trabeculae carnae course over the inner surface of the heart wall, and how the 
papillary muscles are attached to the trabeculae carnae by branching structures at their bases, rather than directly to the solid portion of the heart wall, as has been conventionally believed.

\section{Validation and Conclusion}

The experimental results of our segmentation and reconstruction framework has been qualitatively evaluated by clinical experts. According to them, in most cases our segmentation framework achieves an excellent agreement with the manual segmentation, while the segmentation time is dramatically decreased compared to manual segmentation. More validation results will be available soon to prove the possible clinical usage of this method. We also plan to use the framework on other image modules with different noise levels. Currently, we tried the framework on a 192 by 192 by 18 MRI cardiac data to reconstruct the endocardial surface of the LV. The result has also been approved by clinical experts.

Although the inner surface of the cardiac chambers is well known to be rough, the three-dimensional structure of the endocardial surface has not been previously well demonstrated non-invasively in vivo. In particular, the relationship of the papillary muscles to the heart wall can be seen with our high resolution CT images to be not a simple joining of the base of the papillary muscles with the solid portion of the wall, as has been conventionally believed, but rather a branching connection of the base with the trabeculae carnae lining the ventricular cavity. This has not been appreciated with previous tomographic imaging methods, which have had insufficient resolution to demonstrate these structures and limited 3-D registration between different image planes. Study of ex vivo specimens has also not demonstrated this relationship, due to the typically highly contracted state of these specimens. The ability to efficiently and accurately segment these high resolution 3D CT images for full 3D demonstration of the structural relationships of the interior of the heart should provide us with a valuable new tool for the study of normal and abnormal cardiac anatomy. It should also lead us to new insights as to the functional significance of these anatomical relationships.

Our 3D hybrid segmentation framework has provided high resolution segmentation results of the complex cardiac structure. This is the first time that the roughness on the endocardial surface has been so fully reconstructed in 3D. Its time efficiency should enable us to apply it to more clinical use. The high convergence at concavities shows the advantage of the hybrid framework and the strength of the scalar gradient. Our aim is to make further improvements to the methodology so that it can be used as a standard method for 3D cardiac data segmentation and reconstruction.

\section{References}

1. D.N. Metaxas.: Physics-Based Deformable Models: Application to Computer Vision, Graphics and Medical Imaging. (1996) 
2. Chen, T., Metaxas, D.: Gibbs prior models, marching cubes, and deformable Models: A hybrid framework for 3D medical image segmentation. Proceedings of MICCAI Montreal 6 (2003) 703-710

3. P. K. Saha, J. K. Udupa: Tensor-scale-based fuzzy connectedness image segmentation. SPIE4.23 (2003) 1580-1590

4. T. Cootes, C. Taylor, D. Cooper, J. Graham, Active shape model - their training and application. Computer Vision, Graphics, and Image Process: Image Understanding, 1(61): 38-59, 1994.

5. Jinah Park, Dimitris Metaxas, and Leon Axel, Volumetric deformable models with parameter functions: A new approach to the $3 \mathrm{D}$ motion analysis of the LV from MRI-SPAMM, in Proceedings of International Conference on Computer Vision pp 700-705, 1995.

6. C. Xu and J. L. Prince, Snakes, shapes and gradient vector flow, IEEE Trans. Image Processing, vol7, no. , pp. 359-369, 1998.

7. T. Cootes, G J. Edwards, and C. J. Taylor. Active appearance models. In Proc. of ECCV'98, volume 2, pp 484-498. 1998

8. M. R. Kaus, V. Pekar, C. Lorenz, R. Truyen, S. Lobregt, and J. Weese. Automated 3D PDM construction from segmented images using deformable models. IEEE Trans. Med. Imag., 22(8): 1005-1013, 2003.

9. M. Lorenzo-Valdes, G. I. Sanchez-Ortiz, R. Mohiaddin, and D. Rueckert. Segmentation of $4 \mathrm{D}$ cardiac MR images using a probabilistic atlas and the EM algorithm. In Proc. MICCAI 2003. 440-447

10. S. C. Mitchell, B. P. F. Lelieveldt, R. J. van der Geest, H. G. Bosch, J. H. C. Reiber, and M. Sonka. Multistage hybrid active appearance model matching: Segmentation of left and right ventricles in cardiac MR images. IEEE Trans. Med. Imag., 20(5): 415-423, 2001

11. J. Lotjonen, J. Koikkalainen, D. Smutek, S. Kivisto, and K. Lauerma. Fourchamber 3-D statistical shape model from cardiac short-axis and long axis MR data. MICCAI 2003. pp 459-466.

12. K. Park, D. Metaxas, L. Axel. A finite element model for functional analysis of 4D cardiac-tagged MR images. MICCAI 2003. pp 491-498 